\title{
SDF-1/CXCR4 axis promotes osteogenic differentiation of BMSCs through the JAK2/STAT3 pathway
}

\author{
Wen Xiong, Xin Guo, Xianhua Cai
}

The First School of Clinical Medicine, Southern Medical University, Guangdong, China

\begin{abstract}
Introduction. This study aimed to investigate the effects of stromal cell-derived factor-1 (SDF-1) and activation of its receptor, chemokine receptor 4 (CXCR4), on the osteogenic differentiation of bone marrow mesenchymal stem cells (BMSCs), and the key signaling mechanisms involved in these effects.

Material and methods. BMSCs were treated with $100 \mu \mathrm{g} / \mathrm{L} \mathrm{SDF}-1$ and cultured in osteogenic medium for 7 days. RT-qPCR and western blotting were used to detect the protein and mRNA levels of Janus kinase 2 (JAK2), signal transducer and activator of transcription 3 (STAT3), Runt-related transcription factor 2 (Runx2), and osteocalcin $(\mathrm{OCN})$. Alizarin-red staining was used to detect the mineralization-inducing ability of the cells.

Results. After BMSCs were treated with SDF-1, the levels of JAK2 mRNA, STAT3 mRNA, and protein phosphorylation increased, the number of mineralized nodules of BMSCs increased, and the osteogenic-differentiation ability was enhanced. In addition, after BMSCs were treated with an inhibitor of JAK2 phosphorylation, the levels of JAK2, STAT3, Runx2, and OCN decreased significantly, the number of mineralized nodules of BMSCs also decreased, and the osteogenic-differentiation ability decreased. The inhibition of CXCR4-treated BMSCs further confirmed that SDF-1/CXCR4 activated JAK2/STAT3 to regulate the osteogenic differentiation of BMSCs. Conclusions. SDF-1/CXCR4 promoted the osteogenic differentiation of BMSCs through JAK2/STAT3 activation. (Folia Histochemica et Cytobiologica 2021, Vol. 59, No. 3, 187-194)
\end{abstract}

Key words: BMSCs; osteogenic differentiation; SDF-1; CXCR4; JAK2/STAT3; Runx2, osteocalcin; alizarin red

\section{Introduction}

Bone marrow mesenchymal stem cells (BMSCs) are non-hematopoietic stem cells present in the bone marrow. BMSCs cultured in vitro can adhere to the vessel wall and proliferate rapidly, exhibiting a fibroblast-like long fusiform shape. BMSCs have the potential for cross-system and cross-germ differentiation under the action of a specific microenvironment and suitable cytokines/growth factors. They can differentiate into mesodermal and neuroectodermal cells, such as osteoblasts, chondrocytes, endothelial cells, and nerve cells;

\footnotetext{
Correspondence address: Xianhua Cai

The First School of Clinical Medicine,

Southern Medical University,

No.1023, South Shatai Road, Baiyun District,

Guangzhou 510515, Guangdong, China

e-mail:whcaixh@163.com
}

accordingly, they have recently become a resource for tissue repair [1, 2]. Osteogenic differentiation of BMSCs is closely related to bone-related diseases: for example, the inhibition of osteoporosis [3]. Chen et al. demonstrated that osteogenic differentiation of BMSCs promotes new bone formation, which in turn, accelerates fracture healing [4]. Therefore, it is important to study the molecular mechanisms related to the differentiation of BMSCs.

Stromal cell-derived factor-1 (SDF-1) is a chemokine protein. It mainly binds to $\mathrm{CXC}$ receptor 4 (CXCR4) and plays an important role in the homeostasis of organ development and hematopoietic cell differentiation $[5,6]$. The interaction between SDF-1 and CXCR4 can control the migration, osteogenic differentiation, and survival of BMSCs under oxidative stress [7-11]. SDF-1 has also been shown to accelerate and enhance calcium deposition, regulate a variety of osteogenic factors such as runt-related 
transcription factor 2 (Runx2), bone morphogenetic protein 2 (BMP2), and osteocalcin (OCN), enhance alkaline phosphatase activity, and maintain bone homeostasis by assisting BMSCs to migrate to injured tissues [12,13]. However, how the SDF-1/CXCR4 axis regulates the osteogenic differentiation of BMSCs remains unclear.

Janus kinase 2/signal transducer and activator of transcription 3 (JAK2/STAT3) signaling in osteoblasts plays an important role in bone biology [14]. By contrast, activation of JAK2/STAT3 signaling in BMSCs has been reported to inhibit bone regeneration [15]. Therefore, the exact role of JAK2/STAT3 signaling in bone biology remains controversial. The collection, proliferation, and osteogenic differentiation of mesenchymal stem cells are important for the treatment of bone defects [16]. In recent reports, JAK2/STAT3 signaling plays an important role in the proliferation and osteogenic differentiation of BMSCs [17], and SDF-1 can induce the activation of STAT3 and improve the migration of bone marrow stromal cells in a middle cerebral artery occlusion stroke model [18]. Nevertheless, whether SDF-1/CXCR4 can induce BMSC differentiation by promoting JAK2/STAT3 pathway activation has not been reported. In view of the current research, this study aimed to explore the effect of SDF-1 and the activation of its receptor, CXCR4, on the osteogenic differentiation of bone marrow mesenchymal stem cells (BMSCs) and the key signaling mechanism.

\section{Materials and methods}

Cell culture. Human bone marrow mesenchymal stem cells (hBMSCs) were purchased from ScienCell (San Diego, CA, USA) and cultured in -MEM (HyClone, Logan, UT, USA) containing $15 \% \mathrm{FBS}, 100 \mu \mathrm{M}$ L-ascorbic acid, $2 \mathrm{mM} \mathrm{L-glu-}$ tamine, $100 \mathrm{U} / \mathrm{mL}$ penicillin, and $100 \mu \mathrm{g} / \mathrm{mL}$ streptomycin (Yaanda Biotechnology Co., Ltd., Beijing, China), at $37^{\circ} \mathrm{C}$ in a $5 \% \mathrm{CO}_{2}$ cell culture chamber.

SDF-1 treatment and cell grouping. The cells $\left(1 \times 10^{5}\right.$ cells $/ \mathrm{mL}$ ) were cultured in 12-well plates with osteogenic differentiation medium $(\alpha$-MEM $+0.1 \mu \mathrm{M}$ dexamethasone $+10 \mathrm{mM} \beta$-glycerophosphate $+50 \mu \mathrm{g} / \mathrm{mL}$ ascorbic acid) to induce osteogenic differentiation of hBMSCs for 7 days. The experiment was divided into two groups: the control group (cultured in osteogenic differentiation medium for 7 days), and the SDF-1 (PeproTech, East Windsor, NJ, USA) induced group ( $100 \mu \mathrm{g} / \mathrm{LSDF}-1$ was added to the cells for $30 \mathrm{~min}$ and the cells were washed with phosphate-buffered saline (PBS) which were then cultured in osteogenic differentiation medium for 7 days). Cells need to be washed with culture medium after replacing the medium after addition of drugs.
AG490 treatment and cell grouping. The experiment was divided into four groups: the control group, the AG490 (JAK2 phosphorylation inhibitor, MCE, China) group (cells were treated with $50 \mu \mathrm{mol} / \mathrm{L} \mathrm{AG490}$ for $72 \mathrm{~h}$ and cultured in osteogenic differentiation medium for 7 days), the SDF-1 group $(100 \mu \mathrm{g} / \mathrm{L} \mathrm{SDF}-1$ was added to the cells for $30 \mathrm{~min}$ which were then cultured in osteogenic differentiation medium for 7 days), the SDF-1 + AG490 group (the cells were treated with $100 \mu \mathrm{g} / \mathrm{L} \mathrm{SDF}-130 \mathrm{~min}$, and with $50 \mu \mathrm{mol} / \mathrm{L}$ AG490 for $72 \mathrm{~h}$ and cultured in osteogenic differentiation medium for 7 days). Cells need to be washed with culture medium after replacing the medium after addition of drugs.

AMD3100 treatment and cell grouping. The experiment was divided into four groups: the control group, the AMD3100 (SDF-1/CXCR4 receptor antagonist, MCE, China) group $(5 \mu \mathrm{g} / \mathrm{mL}$ AMD3100 were added to the cell culture for $48 \mathrm{~h}$ and the cells were cultured in osteogenic differentiation medium for 7 days), the SDF-1 group (100 $\mu \mathrm{g} / \mathrm{L} \mathrm{SDF-1} \mathrm{was}$ added for $30 \mathrm{~min}$ and the cells were cultured in osteogenic differentiation medium for 7 days), the SDF-1 + AMD3100 group $(100 \mu \mathrm{g} / \mathrm{L}$ SDF-1 was added for $30 \mathrm{~min}, 5 \mu \mathrm{g} / \mathrm{mL}$ AMD3100 was added for $48 \mathrm{~h}$ and the cells were cultured in osteogenic differentiation medium for 7 days). Cells need to be washed with culture medium after replacing the medium after addition of drugs.

RT-qPCR. Total RNA was extracted using TRIzol reagent (Invitrogen, Carlsbad, CA, USA), and then DNA was removed using a turbo DNase Kit (Ambion). The extracted RNA was quantified using a NanoDrop spectrophotometer. A Primescript real-time (RT) kit (Takara Bio, Shiga, Japan) was used to synthesize complementary DNA using $1000 \mathrm{ng}$ of total RNA. SYBR Select Master Mix (Applied Biosystems, Waltham, MA, USA) was used to perform quantitative real-time PCR on an Applied Biosystems 7900HT system. Glyceraldehyde 3-phosphate dehydrogenase (GAPDH) was used as the standardized internal reference for JAK2, STAT3, Runx2, and OCN mRNA. The CT value was calculated using the $2^{-\Delta \Delta \mathrm{Ct}}$ method. The primer sequences used in this study were as follows:

JAK2, F:5'-TCTGGGGAGTATGTTGCAGAA-3', R:5'-AGACATGGTTGGGTGGATACC-3';

STAT3, F:5'-CAGCAGCTTGACACACGGTA-3', R:5'-AAACACCAAAGTGGCATGTGA-3';

Runx2, F:5'-CGCGTTGCATAGTCACAAAA-3', R:5'-AGTGCAGGGTCCGAGGTATT-3';

OCN, F:5'-CACTCCTCGCCCTATTGGC-3', R:5'-CCCTCCTGCTTGGACACAAAG-3';

GAPDH, F:5'-TGTGGGCATCAATGGATTTGG-3', R:5'-CCCTCCTGCTTGGACACAAAG-3'.

Western blotting. Total cellular proteins were extracted using a radioimmunoprecipitation assay (RIPA; Beyotime, 


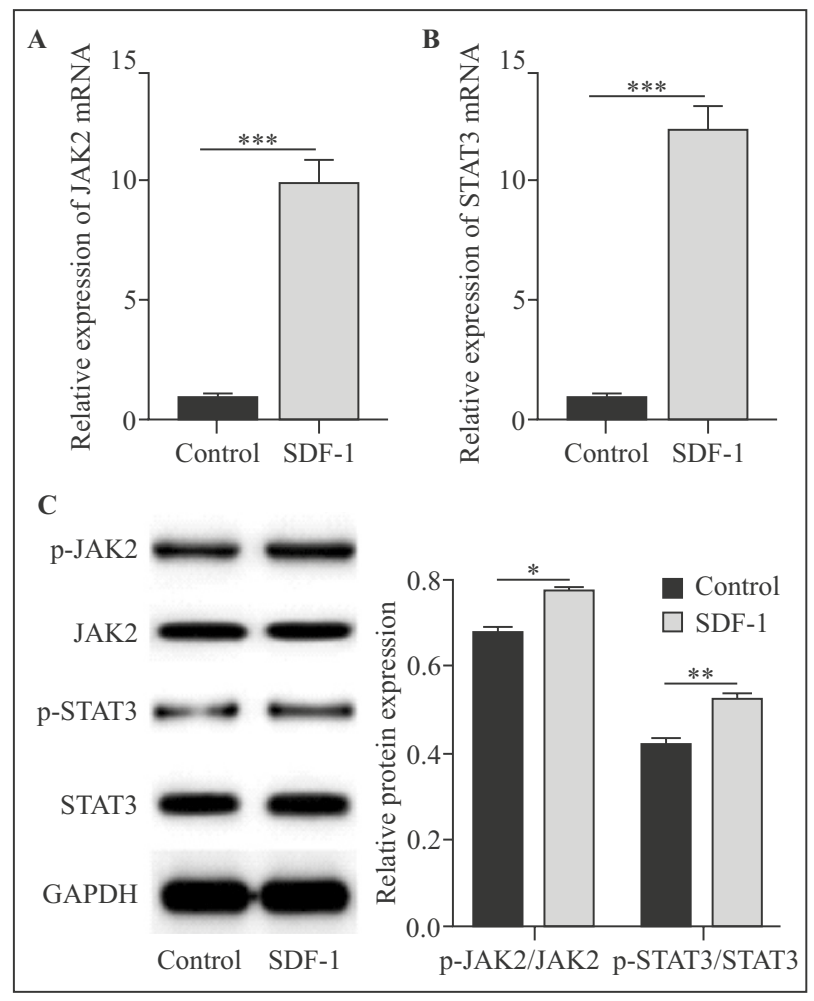

Figure 1. Effect of SDF-1 on JAK2/STAT3 mRNA and protein levels of SDF-1-treated BMSCs. BMSCs were treated with SDF-1 (100 $\mu \mathrm{g} / \mathrm{L})$ for $30 \mathrm{~min}$ (A and B) and cells were collected after 7 days. The mRNA levels of JAK2 and STAT3 were detected by RT-qPCR. Western blotting was used to detect the levels of the JAK2/STAT3-related proteins: p-JAK2, JAK2, p-STAT3, and STAT3. Data are means of three independent experiments, and each independent experiment was repeated with three replicate wells. ${ }^{*} P<0.05,{ }^{* *} P<0.01,{ }^{* * *} P<0.001 v$ s. control group.

Shanghai, China). The total protein was separated by electrophoresis and transferred to a polyvinylidene fluoride (PVDF) membrane (Millipore, Billerica, MA, USA). After blocking with $5 \%$ skim milk for $2 \mathrm{~h}$, the membrane was incubated with the primary antibodies - Anti-Runx2 (ab192256, 1:1000, Abcam, Cambridge, UK), anti-OCN (ab270202, 1:1000, Abcam), anti-JAK2 (ab108596, 1:1000, Abcam), anti-STAT3 (ab68153, 1:1000, Abcam) at $4^{\circ} \mathrm{C}$ overnight. The next day, the membrane was incubated with the corresponding secondary antibody at room temperature for $2 \mathrm{~h}$. Immune response bands were exposed to enhanced chemiluminescence (ECL; Thermo Fisher Science, Waltham, MA, USA) and analyzed using ImageJ software (NIH, Bethesda, MD, USA).

Alizarin-red staining. After 7 days, the cells from the six-well plates of each group were collected. The cells were removed from the culture medium, then washed twice with PBS. The cells were fixed with $10 \%$ formaldehyde at room temperature for $15 \mathrm{~min}$. The cells were rinsed twice with redistilled water, and $40 \mathrm{mM}$ alizarin red dye solution was added, $1 \mathrm{~mL}$ per well. The cells were incubated at room temperature for $20 \mathrm{~min}$ and slightly oscillated. To remove dyes that were not fully bound, the cells were rinsed with redistilled water and shaken for $5 \mathrm{~min}$; this was repeated four times. Excess redistilled water was allowed to absorb before observation under an inverted microscope.

Statistical analysis. GraphPad Prism 8 (GraphPad Inc., La Jolla, CA, USA) was used for statistical analysis. The differences between experimental groups were analyzed by Student's $t$-test. Single-factor analysis of variance (ANOVA) was used for comparison among groups and then a minimum-significant-difference test was performed. $P<0.05$ was considered to be statistically significant.

\section{Results}

\section{Effect of SDF-1 on JAK2/STAT3 levels in BMSCs}

To explore the effect of the SDF-1/CXCR4 axis on BMSCs, BMSCs were treated with SDF-1 $(100 \mu \mathrm{g} / \mathrm{L})$. The levels of the JAK2/STAT3-related proteins p-JAK2, JAK2, p-STAT3, and STAT3 - and their mRNAs were detected by RT-qPCR and Western blotting. The results showed that the levels of JAK2 mRNA, STAT3 mRNA, and their protein phosphorylation in the SDF-1 treated group were higher than those of the non-SDF-1 group ( $p<0.05$, Fig. 1A-C). 


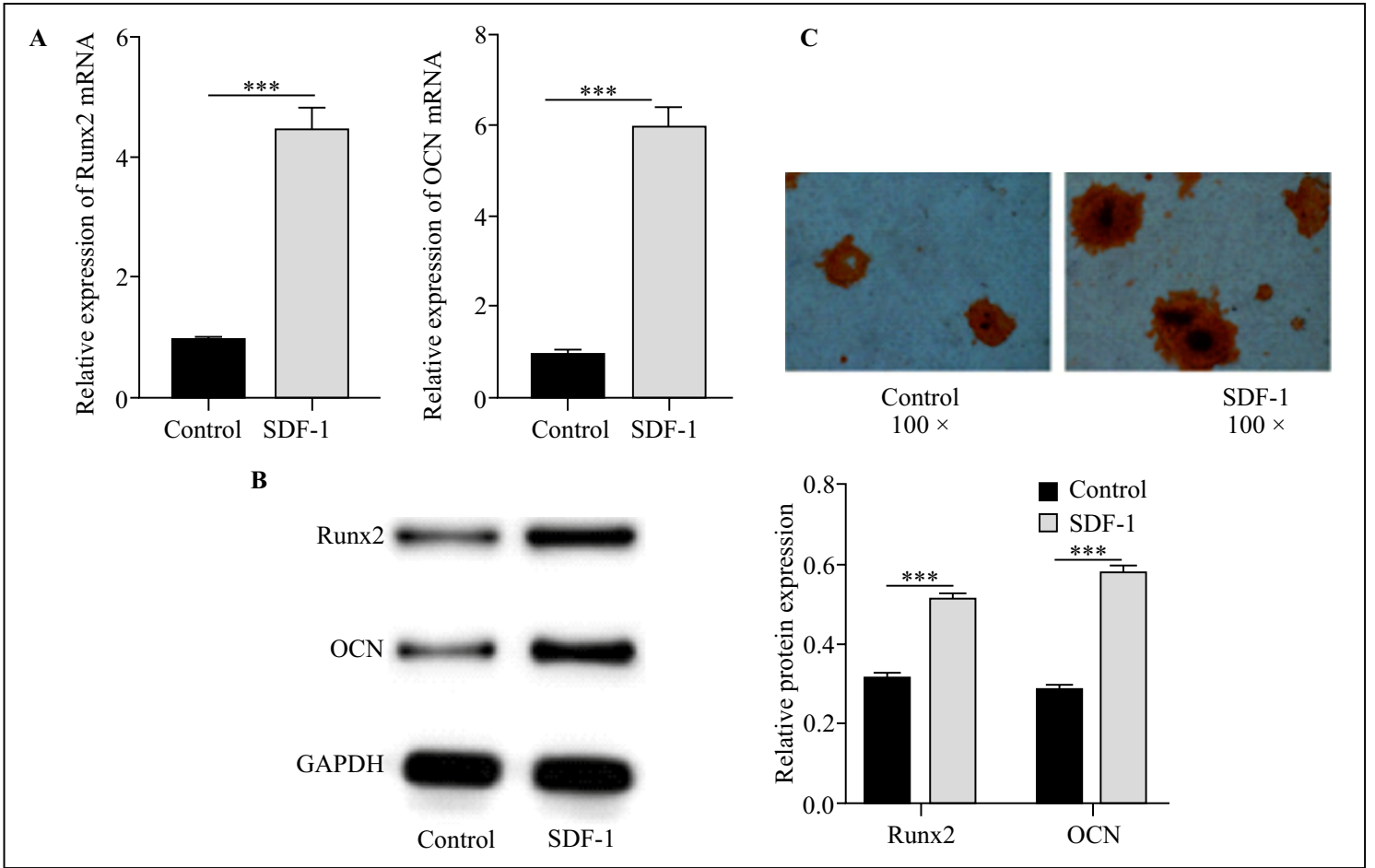

Figure 2. SDF-1 regulated osteogenic differentiation of BMSCs. BMSCs were treated with SDF-1 (100 $\mu \mathrm{g} / \mathrm{L})$ for $30 \mathrm{~min}$ and cells were collected after 7 days. A. The mRNA levels of Runx2 and osteocalcin (OCN) were detected by RT-qPCR. B. The levels of Runx 2 and $\mathrm{OCN}$ were detected by western blotting. C. Alizarin-red staining assay used to detect the mineralization-induction ability of the cells. Data are means of three independent experiments, and each independent experiment was repeated with three replicate wells. ${ }^{* *} P<0.001 v s$. control group.

Thus, the results showed that SDF-1 promoted the activation of JAK2/STAT3 in BMSCs.

\section{SDF-1 regulated osteogenic differentiation of BMSCs}

Next, we explored the effect of SDF-1/CXCR4 on the osteogenic differentiation of BMSCs. RT-qPCR and Western blotting were used to detect the expression of the osteogenesis-related genes - Runx2 and OCN. The results showed that, compared with the control group, the expression levels of Runx2, OCN, and their mRNA were significantly upregulated. In addition, the results of alizarin-red staining showed that the number of mineralized nodules and the osteogenic-differentiation ability of BMSCs in the SDF1-treated cells were higher than in the control cells. These results suggested that SDF-1/CXCR4 could promote the osteogenic differentiation of BMSCs $(p<0.05$, Fig. 2A-C).

\section{JAK2/STAT3 participated in SDF-1-mediated osteogenic differentiation of BMSCs}

JAK2/STAT3 signaling has been shown to play an important role in the proliferation and osteogenic differentiation of BMSCs [13]. However, whether JAK2/ /STAT3 is involved in SDF-1-mediated osteogenic differentiation of BMSCs remains unclear. BMSCs were treated with the JAK2 phosphorylation inhibitor, AG490. First, JAK2/STAT3-related protein and mRNA levels were detected by RT-qPCR and western blotting; the results showed that the levels of JAK2, STAT3 mRNA, and the phosphorylation of their proteins in the AG490 group were significantly lower than in the SDF-1 group ( $p<0.05$, Fig. 3A-C). We then explored the effect of the JAK2 phosphorylation inhibitor, AG490, on the osteogenic differentiation of BMSCs. RT-qPCR and western blot results showed that Runx2, OCN, and their mRNA levels were significantly downregulated in the SDF-1 + AG490 group compared with the SDF-1 group $(p<0.05$, Fig. 3D-F). Alizarin-red staining showed that the number of mineralized nodules and osteogenic-differentiation ability of BMSCs in the AG490 group were lower than in the SDF-1 group ( $p<0.05$, Fig. 3G).

\section{SDF-1/CXCR4 activated JAK2/STAT3 pathway to regulate osteogenic differentiation of BMSCs}

To further verify the role of SDF-1/CXCR4 in the osteogenic differentiation of BMSCs by activating JAK2/STAT3, we treated BMSCs with the CXCR4 inhibitor, AMD3100. The results of western blotting 


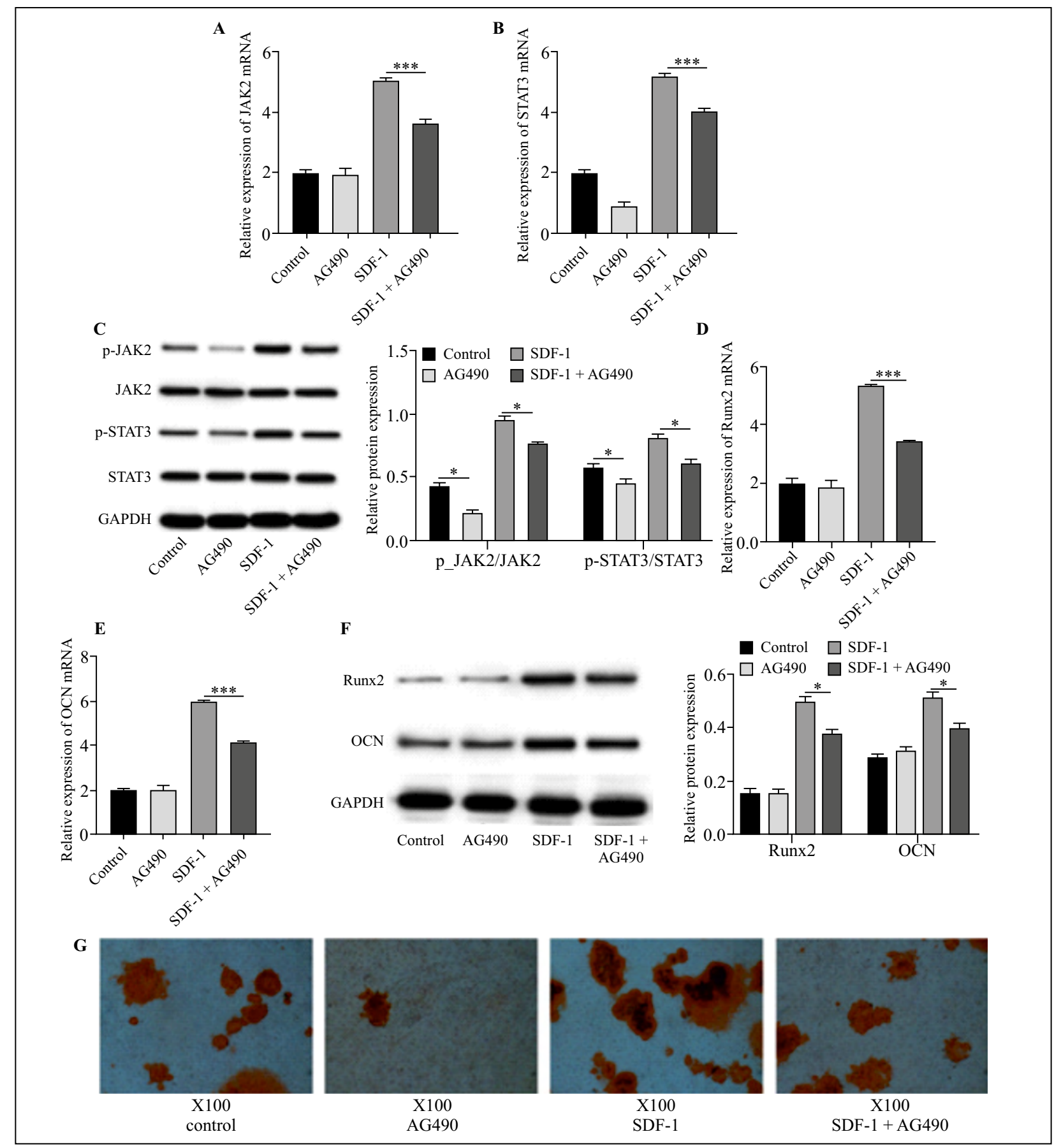

Figure 3. JAK2/STAT3 participated in SDF-1-mediated osteogenic differentiation of BMSCs. BMSCs were treated first with SDF-1 $(100 \mu \mathrm{g} / \mathrm{L})$ for $30 \mathrm{~min}$ and then with the JAK2 phosphorylation inhibitor, AG490 $(5 \mu \mathrm{g} / \mathrm{mL})$ for $72 \mathrm{~h}$ and cells were collected after 7 days. A. and B. Levels of JAK2 mRNA and STAT3 mRNA were detected by RT-qPCR. C. Western blotting was used to detect the levels of JAK2/STAT3-related proteins p-JAK2, JAK2, p-STAT3, and STAT3. D. and E. RT-qPCR was used to detect the levels of Runx 2 mRNA and OCN mRNA. F. The levels of Runx2 and OCN were detected by Western blotting. G. Alizarin-red staining assay was used to detect the ability of cells to induce mineralization. Data are means of three independent experiments, and each independent experiment was repeated with three replicate wells. ${ }^{*} P<0.05,{ }^{* * *} P<0.001$ compared with SDF-1 group.

showed that the phosphorylation of JAK2 and STAT3 proteins was downregulated compared with the control group. In the SDF-1-treated group, AMD3100 treatment reversed the effect of SDF-1 on JAK2 and
STAT3 protein phosphorylation ( $p<0.05$, Fig. 4A). Taken together, these results suggest that SDF-1/ /CXCR4 activated JAK2/STAT3 to regulate the osteogenic differentiation of BMSCs (Fig. 4B). 


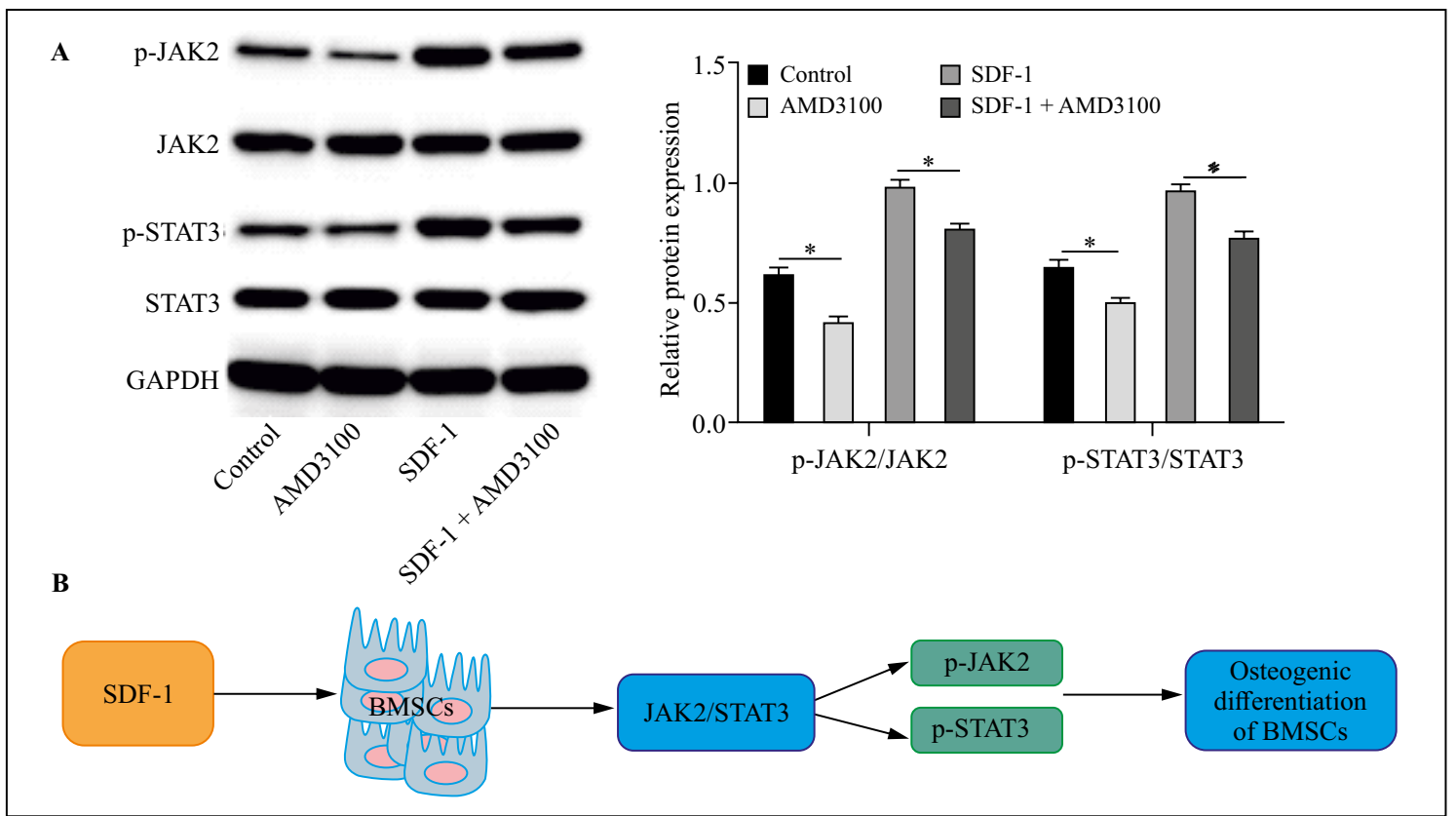

Figure 4. SDF-1/CXCR4 activated JAK2/STAT3 pathway to regulate osteogenic differentiation of BMSCs. BMSCs were first with SDF-1 (100 $\mu \mathrm{g} / \mathrm{L})$ for $30 \mathrm{~min}$ and then exposed for $48 \mathrm{~h}$ to the CXCR4 inhibitor AMD3100 $(5 \mu \mathrm{g} / \mathrm{mL})$, afterwards the cells were collected after 7 days. A. The levels of p-JAK2, JAK2, p-STAT3, and STAT3 were detected by Western blotting. B. The diagram shows postulated mechanisms of SDF-1-induced osteogenic differentiation of BMSCs by the promotion of the SDF-1/CXCR4 activated JAK2/STAT3 signaling. Data are means of three independent experiments, and each independent experiment was repeated with three replicate wells. ${ }^{*} P<0.05$, compared to the control or SDF-1 group.

\section{Discussion}

JAK2 is a non-receptor tyrosine kinase member of the Janus kinase (JAKs) family, which serves as a cytoplasmic signaling component of cytokine receptors and is activated by cytokine-mediated trans-phosphorylation, leading to receptor phosphorylation and recruitment and phosphorylation of signal transducer and activator of transcription (STAT) proteins [19]. As a downstream substrate of JAK2, STAT3 is an inducible monomer transcription factor. After recruitment by JAK2, STAT3 dimerizes and translocates to the nucleus after Tyr705 phosphorylation. The activation of STAT3 signaling can induce changes in the expression of downstream molecules and regulate biological processes such as cell migration, proliferation, and apoptosis [20]. Previous studies have shown that the JAK2/STAT3 pathway is involved in the progression of colorectal cancer [18] and central nervous system diseases related to experimental cerebral ischemia [21]. There are also relevant reports on bone defect diseases. For example, Yu et al. [13] confirmed that inhibition of JAK2/STAT3 signal transduction can inhibit the proliferation and osteogenic differentiation of bone-marrow stromal cells and impede the healing of bone defects, indicating that JAK2/STAT3 plays an important role in the proliferation and osteogenesis of bone marrow stromal cells. AG490, the inhibitor of JAK2, can reduce the phosphorylation of STAT3 by selectively inactivating JAK2. The therapeutic potential of AG490 has been demonstrated for experimental bone-defect in a mouse model [22]. Similarly to the latter research, we used JAK2 phosphorylation inhibitor AG490 to explore the role of JAK2/STAT3 in osteogenic differentiation of BMSCs. The results showed that phosphorylation of JAK2 and STAT3 decreased in BMSCs, and the osteogenic-differentiation ability of BMSCs also decreased. Our results suggest that inhibition of the JAK2/STAT3 pathway could inhibit the osteogenic differentiation of BMSCs.

SDF-1 is a low-molecular-weight chemokine protein, which is the most effective chemokine for bone marrow cells, and belongs to the CXC chemokine family [23]. SDF-1 is continuously secreted by stromal cells. CXCR4 is the only known receptor of SDF1 that belongs to the G-protein-coupled receptor family. SDF-1 and CXCR4 constitute the SDF-1/ /CXCR4 axis, which transduces specific signals and mediates different effects [24]. It plays an important role in embryonic development, mediating immune and inflammatory responses, and regulating stemcell migration and homing [25, 26]. CXCR4 is barely 
expressed in normal tissues but increases in ischemia [27]. CXCR4 is expressed in BMSCs, and cytokines produced in result of tissue injury can stimulate CXCR4 to be transferred from within cells to the cell surface [28]. SDF-1 has a strong chemoattractant effect on CXCR4-expressing BMSCs since the specific binding of SDF-1 to CXCR4 is conducive to the directional chemotaxis of BMSCs [29]. In this study, we found that SDF-1 promotes the osteogenic differentiation of BMSCs.

As a specific inhibitor of CXCR4, AMD3100 has been widely used in basic research on CXCR4 pathway disorders. For example, in myeloma, AMD3100 blocks the interaction between multiple myeloma cells and the bone marrow microenvironment, and enhances multiple myeloma cells' sensitivity to treatment [30]. In this study, to verify the effect of SDF-1/ /CXCR4 regulating JAK2/STAT3 pathway in the osteogenic differentiation of BMSCs, we treated BMSCs with the JAK2 phosphorylation inhibitor AG490 and the CXCR4 specific inhibitor AMD3100. The results showed that AMD3100 treatment could reduce the phosphorylation levels of JAK2 and STAT3. The results further indicated that SDF-1/ CXCR4 could signal through JAK2/ and STAT3 pathway.

In conclusion, our results suggest that SDF-1/ /CXCR4 activates JAK2/STAT3 to regulate the osteogenic differentiation of BMSCs; however, there are still some shortcomings in this study. Xiu et al. [31] confirmed that SDF-1/CXCR4 can promote the migration of BMSCs and enhance SDF-1/CXCR4 therapeutic effect of BMSCs in LPS-induced acute liver injury, which is caused by the activation of the PI3K/ /Akt signaling pathway. Therefore, it is important to study the effect of SDF-1/CXCR4 on other aspects of BMSCs function, including their migration. In addition, previous studies have confirmed that SDF-1/ /CXCR4 reduces adhesion-mediated chemoresistance of multiple myeloma cells by activating PI3K/Akt and upregulating interleukin-6 (IL-6) [32]. Therefore, the role of SDF-1/CXCR4 and other signaling pathways in BMSCs needs to be further explored.

\section{Funding}

None.

\section{Authors' contribution}

CXH: conceived and designed the experiments; WX: performed the experiments; XG: statistic analysis; WX: wrote the paper. All authors read and approved the final manuscript.

\section{Data availability statement}

The data used to support the findings of this study are available from the corresponding author upon request.

\section{Competing interests}

The authors declare that they have no competing interest.

\section{References}

1. Nishigaki A, Tsuzuki-Nakao T, Kido T, et al. Concentration of stromal cell-derived factor-1 (SDF-1/CXCL12) in the follicular fluid is associated with blastocyst development. Reprod Med Biol. 2019; 18(2): 161-166, doi: 10.1002/rmb2.12259, indexed in Pubmed: 30996679.

2. Kawaguchi N, Zhang TT, Nakanishi T. Involvement of CXCR4 in normal and abnormal development. Cells. 2019; 8(2), doi: 10.3390/cells8020185, indexed in Pubmed: 30791675.

3. Li G, An J, Han X, et al. Hypermethylation of microRNA-149 activates SDF-1/CXCR4 to promote osteogenic differentiation of mesenchymal stem cells. J Cell Physiol. 2019; 234(12): 23485-23494, doi: 10.1002/jcp.28917, indexed in Pubmed: 31206187.

4. Chen Q, Zheng C, Li Y, et al. Bone targeted delivery of SDF-1 via alendronate functionalized nanoparticles in guiding stem cell migration. ACS Appl Mater Interfaces. 2018; 10(28): 23700-23710, doi: 10.1021/acsami.8b08606, indexed in Pubmed: 29939711.

5. Li G, Yun X, Ye K, et al. Long non-coding RNA-H19 stimulates osteogenic differentiation of bone marrow mesenchymal stem cells via the microRNA-149/SDF-1 axis. J Cell Mol Med. 2020; 24(9): 4944-4955, doi: 10.1111/jcmm.15040, indexed in Pubmed: 32198976.

6. Zhuang XM, Zhou B, Yuan KF. Role of p53 mediated miR$23 \mathrm{a} / \mathrm{CXCL} 12$ pathway in osteogenic differentiation of bone mesenchymal stem cells on nanostructured titanium surfaces. Biomed Pharmacother. 2019; 112: 108649, doi: 10.1016/j.biopha.2019.108649, indexed in Pubmed: 30784930.

7. Barzelay A, Weisthal Algor S, Niztan A, et al. Adipose-derived mesenchymal stem cells migrate and rescue rpe in the setting of oxidative stress. Stem Cells Int. 2018; 2018: 9682856 , doi: 10.1155/2018/9682856, indexed in Pubmed: 30651740.

8. Herberg S, Fulzele S, Yang N, et al. Stromal cell-derived factor- $1 \beta$ potentiates bone morphogenetic protein-2-stimulated osteoinduction of genetically engineered bone marrow-derived mesenchymal stem cells in vitro. Tissue Eng Part A. 2013; 19(1-2): 1-13, doi: 10.1089/ten.TEA.2012.0085, indexed in Pubmed: 22779446.

9. Periyasamy-Thandavan S, Burke J, Mendhe B, et al. MicroRNA-141-3p negatively modulates SDF-1 expression in age-dependent pathophysiology of human and murine bone marrow stromal cells. J Gerontol A Biol Sci Med Sci. 2019; 74(9): 1368-1374, doi: 10.1093/gerona/gly186, indexed in Pubmed: 31505568.

10. Itoh $\mathrm{S}, \mathrm{Udagawa} \mathrm{N}$, Takahashi $\mathrm{N}$, et al. A critical role for interleukin-6 family-mediated Stat 3 activation in osteoblast differentiation and bone formation. Bone. 2006; 39(3): 505512, doi: 10.1016/j.bone.2006.02.074, indexed in Pubmed: 16679075 .

11. Yue R, Zhou BoO, Shimada IS, et al. Leptin receptor promotes adipogenesis and reduces osteogenesis by regulating 
mesenchymal stromal cells in adult bone marrow. Cell Stem Cell. 2016; 18(6): 782-796, doi: 10.1016/j.stem.2016.02.015, indexed in Pubmed: 27053299.

12. Pajarinen J, Lin T, Gibon E, et al. Mesenchymal stem cell-macrophage crosstalk and bone healing. Biomaterials. 2019; 196: 80-89, doi: 10.1016/j.biomaterials.2017.12.025, indexed in Pubmed: 29329642.

13. Yu X, Li Z, Wan Q, et al. Inhibition of JAK2/STAT3 signaling suppresses bone marrow stromal cells proliferation and osteogenic differentiation, and impairs bone defect healing. Biol Chem. 2018; 399(11): 1313-1323, doi: 10.1515/hsz-20180253, indexed in Pubmed: 30044759.

14. Han J, Feng Z, Xie Yu, et al. Oncostatin M-induced upregulation of SDF-1 improves bone marrow stromal cell migration in a rat middle cerebral artery occlusion stroke model. Exp Neurol. 2019; 313: 49-59, doi: 10.1016/j.expneurol.2018.09.005, indexed in Pubmed: 30213507.

15. Zhang P, Zhang H, Lin J, et al. Insulin impedes osteogenesis of BMSCs by inhibiting autophagy and promoting premature senescence via the TGF- 1 pathway. Aging (Albany NY). 2020; 12(3): 2084-2100, doi: 10.18632/aging.102723, indexed in Pubmed: 32017705.

16. Li X, Zheng Y, Hou L, et al. Exosomes derived from maxillary BMSCs enhanced the osteogenesis in iliac BMSCs. Oral Dis. 2020; 26(1): 131-144, doi: 10.1111/odi.13202, indexed in Pubmed: 31541596.

17. Onodera K, Sakurada A, Notsuda H, et al. Growth inhibition of KRASand EGFRmutant lung adenocarcinoma by cosuppression of STAT3 and the SRC/ARHGAP35 axis. Oncol Rep. 2018; 40(3): 1761-1768, doi: 10.3892/or.2018.6536, indexed in Pubmed: 30015929.

18. Park SY, Lee CJ, Choi JH, et al. The JAK2/STAT3/CCND2 Axis promotes colorectal cancer stem cell persistence and radioresistance. J Exp Clin Cancer Res. 2019; 38(1): 399, doi: 10.1186/s13046-019-1405-7, indexed in Pubmed: 31511084.

19. Hubbard SR. Mechanistic insights into regulation of JAK2 tyrosine kinase. Front Endocrinol (Lausanne). 2017; 8: 361, doi: 10.3389/fendo.2017.00361, indexed in Pubmed: 29379470.

20. Liu H, Du T, Li C, et al. STAT3 phosphorylation in central leptin resistance. Nutr Metab (Lond). 2021; 18(1): 39, doi: 10.1186/s12986-021-00569-w, indexed in Pubmed: 33849593.

21. Chen XM, Yu YH, Wang L, et al. Effect of the JAK2/STAT3 signaling pathway on nerve cell apoptosis in rats with white matter injury. Eur Rev Med Pharmacol Sci. 2019; 23(1): 321-327, doi 10.26355/eurrev_201901_16779, indexed in Pubmed: 30657573.

22. Yu X, Wan QL, Li Z, et al. [(*)AG490 could suppress bone marrow mesenchymal stem cells migration, mineralization and bone defect healing via inhibiting Jak2-STAT3 pathway]. Zhonghua Kou Qiang Yi Xue Za Zhi. 2018; 53(5): 293-300, doi: 10.3760/cma.j.issn.1002-0098.2018.05.002, indexed in Pubmed: 29972985.
23. Teicher BA, Fricker SP. CXCL12 (SDF-1)/CXCR4 pathway in cancer. Clin Cancer Res. 2010; 16(11): 2927-2931, doi: 10.1158/1078-0432.CCR-09-2329, indexed in Pubmed: 20484021.

24. Mousavi A. CXCL12/CXCR4 signal transduction in diseases and its molecular approaches in targeted-therapy. Immunol Lett. 2020; 217: 91-115, doi: 10.1016/j.imlet.2019.11.007, indexed in Pubmed: 31747563.

25. Ding Qi, Sun J, Xie W, et al. Stemona alkaloids suppress the positive feedback loop between M2 polarization and fibroblast differentiation by inhibiting JAK2/STAT3 pathway in fibroblasts and CXCR4/PIK/AKT1 pathway in macrophages. Int Immunopharmacol. 2019; 72: 385-394, doi: 10.1016/j.intimp.2019.04.030, indexed in Pubmed: 31030094.

26. Dimova I, Karthik S, Makanya A, et al. SDF-1/CXCR4 signalling is involved in blood vessel growth and remodelling by intussusception. J Cell Mol Med. 2019; 23(6): 3916-3926, doi: 10.1111/jcmm.14269, indexed in Pubmed: 30950188.

27. Ge G, Zhang H, Li R, et al. The function of SDF-1-CXCR4 axis in SP cells-mediated protective role for renal ischemia/ reperfusion injury by SHH/GLI1-ABCG2 pathway. Shock. 2017; 47(2): 251-259, doi: 10.1097/SHK.0000000000000694, indexed in Pubmed: 27454381.

28. Wang G, Zhuo Z, Zhang Q, et al. Transfection of CXCR-4 using microbubble-mediated ultrasound irradiation and liposomes improves the migratory ability of bone marrow stromal cells. Curr Gene Ther. 2015; 15(1): 21-31, doi: 10.2174/156 6523214666141121111220, indexed in Pubmed: 25414029.

29. Jaerve A, Schira J, Müller HW. Concise review: the potential of stromal cell-derived factor 1 and its receptors to promote stem cell functions in spinal cord repair. Stem Cells Transl Med. 2012; 1(10): 732-739, doi: 10.5966/sctm.2012-0068, indexed in Pubmed: 23197665.

30. Azab AK, Runnels JM, Pitsillides C, et al. CXCR4 inhibitor AMD3100 disrupts the interaction of multiple myeloma cells with the bone marrow microenvironment and enhances their sensitivity to therapy. Blood. 2009; 113(18): 4341-4351, doi: 10.1182/blood-2008-10-186668, indexed in Pubmed: 19139079

31. Xiu G, Li X, Yin Y, et al. SDF-1/CXCR4 augments the therapeutic effect of bone marrow mesenchymal stem cells in the treatment of lipopolysaccharide-induced liver injury by promoting their migration through PI3K/Akt signaling pathway. Cell Transplant. 2020; 29: 963689720929992, doi: 10.1177/0963689720929992, indexed in Pubmed: 32452221.

32. Liu Y, Liang HM, Lv YQ, et al. Blockade of SDF-1/CXCR4 reduces adhesion-mediated chemoresistance of multiple myeloma cells via interacting with interleukin-6. J Cell Physiol. 2019; 234(11): 19702-19714, doi: 10.1002/jcp.28570, indexed in Pubmed: 30953364.

Submitted: 18 May, 2021

Accepted after reviews: 24 September, 2021 Available as AoP: 27 September, 2021 